\title{
DISTRIBUIÇÃo E ESTRUTURA DAS ESPÉCIES LENHOSAS EM UMA RESTINGA ECOTONAL NO LITORAL AMAZÔNICO MARANHENSE, BrasiL
}

\author{
DISTRIBUTION AND STRUCTURE OF WOODY SPECIES IN AN ECOTONAL \\ Restinga on the Amazon coast of Maranhão state, Brazil
}

\author{
Luciana Belfort ${ }^{1}\left(\mathbb{D}\right.$, Flávia Raquel Fernandes do Nascimento ${ }^{2}$ y \\ Eduardo Bezerra de Almeida Jr. ${ }^{*}$ (iD
}

1. Programa de Pós-Graduação em Biodiversidade e Conservação, Universidade Federal do Maranhão, Av. dos Portugueses, 1966, São Luís, Maranhão, Brasil

2. Departamento de Patologia, Universidade Federal do Maranhão Av. dos Portugueses, 1966, São Luís, Maranhão, Brasil

3. Departamento de Biologia, PósGraduação em Biodiversidade e Conservação, Universidade Federal do Maranhão Av. dos Portugueses, 1966, São Luís, Maranhão, Brasil.

*ebaj25@yahoo.com.br

\section{Citar este artículo}

BELFORT, L., F. R. FERNANDES DO NASCIMENTO \& E. BEZERRA DE ALMEIDA JR. 2021. Distribuição e estrutura das espécies lenhosas em uma restinga ecotonal no litoral Amazônico Maranhense, Brasil. Bol. Soc. Argent. Bot. 56: 561-574.

DOI: https://doi. org/10.31055/1851.2372.v56. n4.32900

Recibido: 30 Abr 2021

Aceptado: 29 Sep 2021

Publicado en línea: 4 Nov 2021

Publicado impreso: 20 Dic 2021

Editor: Roberto Manuel Salas iD

ISSN versión impresa 0373-580X

ISSN versión on-line 1851-2372

\section{SUMMARY}

Background and aims: Restinga vegetation is an ecosystem that extends along the coast of Brazil in several extensions, with phytophysiognomies composed of strata varying from herbaceous to arboreal. The objective of this work was to analyze the structural arrangement, the diversity and distribution of the woody community of an ecotonal restinga on the coast of Maranhão, Northeast Brazil.

M\&M: For sampling the vegetation, the method of points-quadrants (50 points) was used, whose inclusion criteria was Perimeter at Ground Height $\geq 10 \mathrm{~cm}$. Phytosociological parameters, community structure and phytogeographic distribution pattern were analyzed.

Results: The sampling resulted in 22 species, 18 genera and 14 families. The most representative families were Myrtaceae, Arecaceae, Fabaceae, Ochnaceae, Polygonaceae e Rubiaceae. The species with the highest Importance Values (IV) were Astrocaryum vulgare (20,36\%), Attalea speciosa (19,40\%), Himatanthus drasticus (14,72\%), Chamaecrista ramosa (10,68\%), and Eugenia biflora (10,27\%). The Shannon's diversity index was 2,401 nat.ind $^{-1}$ and Pielou's equality was 0,749 . The pattern of local distribution showed that the species are randomly distributed.

Conclusions: The structure and dynamics of the ecotonal restinga between the Amazon and Northeastern coastlines indicate that the area has richness and diversity of species with values similar to the restingas of the northeast, highlighting the need for protection and valuation of plants in restinga areas.

\section{KEY WORDS}

Importance value, Maranhão state; Northeast of Brazil, phytosociology.

\section{Resumo}

Introdução e objetivo: As restingas são peculiares por se estenderem ao longo do litoral em variadas extensões, com fitofisionomias compostas por estrato variando entre herbáceo a arbóreo. O objetivo deste trabalho foi analisar o arranjo estrutural, a diversidade e a distribuição da comunidade lenhosa de uma restinga ecotonal no litoral maranhense, nordeste do Brasil.

M\&M: Para amostragem da vegetação foi utilizado o método de pontos-quadrantes (50 pontos), cujo critério de inclusão foi Perímetro à Altura do Solo $\geq 10 \mathrm{~cm}$. Foram analisados os parâmetros fitossociológicos, a estrutura da comunidade e o padrão de distribuição fitogeográfica.

Resultados: Foram amostradas 22 espécies, 18 gêneros e 14 famílias. As famílias mais representativas foram Myrtaceae, Arecaceae, Fabaceae, Ochnaceae, Polygonaceae e Rubiaceae. As espécies de maiores Valores de Importância (VI) foram Astrocaryum vulgare (20,36\%), Attalea speciosa (19,40\%), Himatanthus drasticus $(14,72 \%)$, Chamaecrista ramosa (10,68\%) e Eugenia biflora $(10,27 \%)$. $\mathrm{O}$ índice de diversidade de Shannon foi 2,401 nat.ind $^{-1}$ e a equitabilidade de Pielou 0,749 . O padrão de distribuição local mostrou que as espécies estão distribuídas aleatoriamente.

Conclusões: A estrutura e dinâmica da restinga ecotonal entre os litorais amazônico e nordestino indica que a área tem riqueza e diversidade de espécies com valores semelhantes as restingas do nordeste, ressaltando a necessidade de proteção e valoração das plantas das áreas de restinga.

\section{Palavras-Chave}

Fitossociologia; Maranhão; Nordeste; valor de importância. 


\section{INTRODUÇÃO}

A intensa antropização nas regiões costeiras para o desenvolvimento do turismo, especulação imobiliária e instalações de portos, provocam degradação e perdas irreparáveis à diversidade vegetal das restingas, sendo necessária a implementação de efetivas unidades de conservação para proteção deste ambiente (Rocha et al., 2007; Braz et al., 2013). No Brasil, as restingas são peculiares por se estenderem ao longo do litoral em variadas extensões, com fitofisionomias compostas por estrato variando entre herbáceo a arbóreo (Suguio \& Tessler, 1984; Braz et al., 2013).

Considerando a riqueza da flora, a extensão territorial, os riscos de ameaça e a carência de dados ecológicos sobre as restingas, esforços foram intensificados para conhecer a diversidade e estrutura da vegetação litorânea (Zickel et al., 2015). Nesse contexto, estudos fitossociológicos mostram-se importantes por caracterizar a comunidade vegetal (Walter et al., 2015), auxiliar no entendimento do arranjo estrutural dessas comunidades e subsidiar medidas de recuperação, manejo e conservação dos ecossistemas (Velazco et al., 2015; Martins et al., 2017), na costa Norte e Nordeste do Brasil.

Nesta última década, análises sobre a diversidade da comunidade lenhosa de restingas foram realizadas ao longo do litoral nordestino, com destaque para os estados de Pernambuco (Almeida Jr. et al., 2011; Cantarelli et al., 2012; Zickel et al., 2015) e Bahia (Menezes et al., 2009; Silva \& Menezes, 2012; Santos et al., 2015). Todavia, apenas três estudos (Machado \& Almeida Jr., 2019; Almeida Jr. et al., 2020; Paiva \& Almeida Jr., 2020) foram desenvolvidos no litoral maranhense, necessitando de mais pesquisas para ampliar o conhecimento sobre a distribuição das espécies ao longo da costa do Estado do Maranhão, considerada a segunda maior do Nordeste.

Cabe ressaltar que o litoral maranhense se encontra dividido em duas vertentes. A porção oeste, caracterizada como litoral amazônico e a porção leste pertencente ao litoral nordestino setentrional (Villwock et al., 2005). A Ilha do Maranhão encontra-se na divisa entre o litoral amazônico e o nordestino (Silveira, 1964). E, considerando que as restingas são colonizadas por espécies de ecossistemas adjacentes (Scarano, 2002), espera-se que nas restingas maranhenses, no trecho referente ao litoral amazônico se verifique uma maior representação de espécies do domínio Amazônico, e no litoral nordestino um maior registro de espécies do Cerrado. Diante disso, acredita-se que por ser uma região ecotonal, a costa da Ilha do Maranhão apresente características vegetacionais comuns aos litorais amazônico e nordestino, sendo um diferencial quanto à sua composição e distribuição de espécies.

Apesar desta particularidade, pesquisas sobre as áreas ecotonais da restinga da Ilha do Maranhão ainda são escassas quanto ao conhecimento e caracterização do estrato lenhoso (Machado \& Almeida Jr., 2019; Paiva \& Almeida Jr. 2020; Almeida Jr. et al., 2020). Embora existam registros da riqueza florística das restingas maranhenses (Cabral-Freire \& Monteiro, 1993; Serra et al., 2016; Lima \& Almeida Jr., 2018; Correia et al., 2020; Guterres et al., 2020) faz-se necessário compreender a organização e o arranjo estrutural da vegetação.

Neste contexto, o presente trabalho teve como objetivo avaliar o arranjo estrutural da comunidade lenhosa de uma área de restinga ecotonal. Para isso, foram levantadas as seguintes questões: i) Qual a composição estrutural da comunidade lenhosa da restinga de Panaquatira? ii) Qual a diversidade do estrato lenhoso da restinga de Panaquatira? iii) Em quais tipos de vegetação as espécies da restinga de Panaquatira ocorrem?

\section{Material e Métodos}

\section{Área de estudo}

$\mathrm{O}$ estudo foi desenvolvido em uma área de restinga na praia de Panaquatira $\left(02^{\circ} 28^{\prime} 23^{\prime \prime} \mathrm{S}\right.$ 04403'13,8”W, Fig. 1), localizada no município de São José de Ribamar, estado do Maranhão, Brasil. Com extensão de 5,6 km, a praia de Panaquatira apresenta topografia plana com declive suave (Souza et al., 2006), ficando na região de transição entre os dois tipos do litoral brasileiro (Lima e Almeida Jr., 2018), o litoral amazônico e o litoral nordestino setentrional. A região apresenta clima tropical do tipo Aw, conforme a classificação de Köppen (Peel et al., 2007). As temperaturas na Ilha do Maranhão variam ao longo do ano de $25,5{ }^{\circ} \mathrm{C}$ a $28,6^{\circ} \mathrm{C}$, com índice pluviométrico médio em torno $2.000 \mathrm{~mm} / \mathrm{ano}$ (IMESC, 2011). 


\section{Belfort et al. - Vegetação lenhosa em uma restinga no litoral Amazônico}
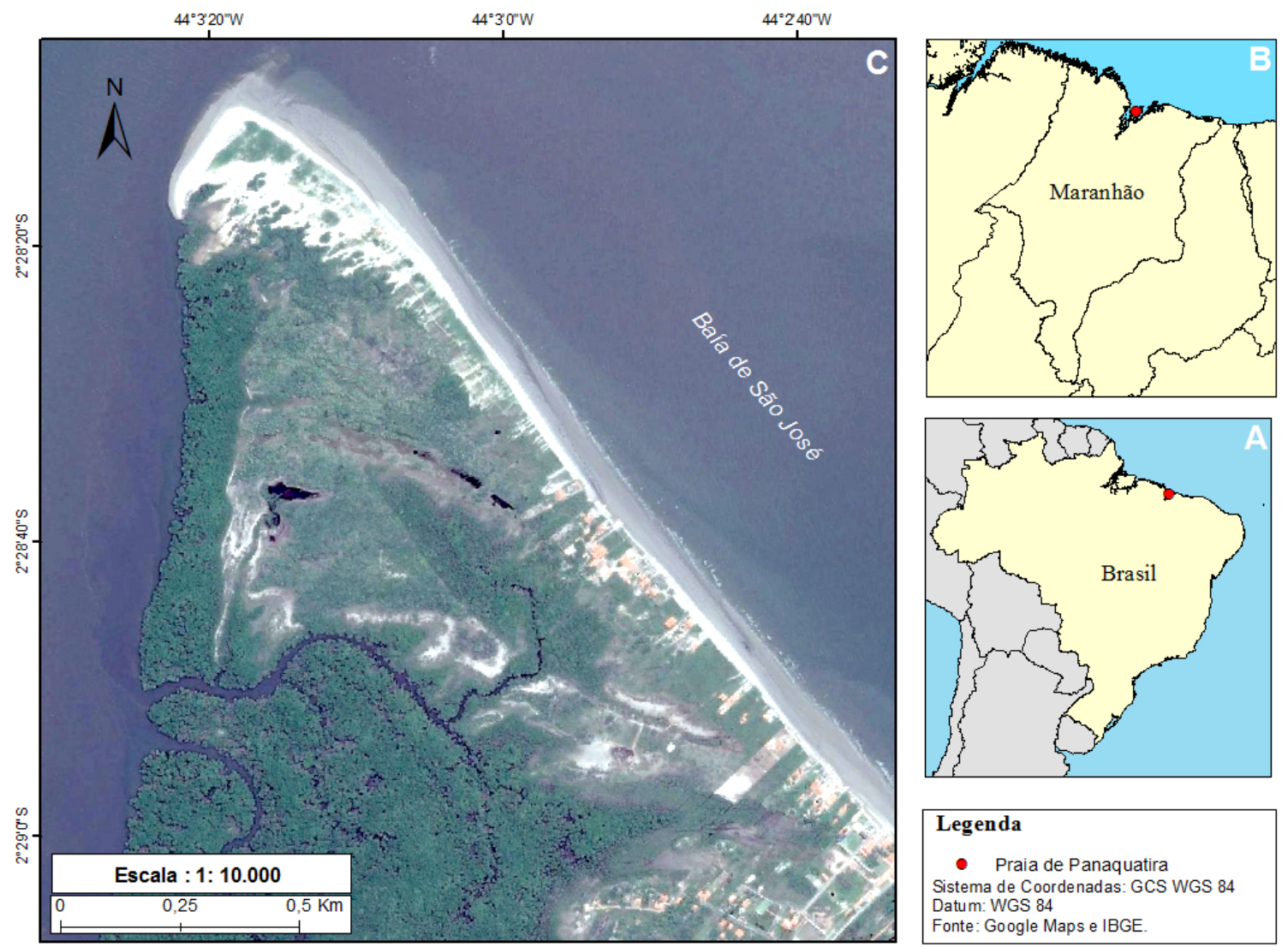

\section{Legenda}

- Praia de Panaquatira

Sistema de Coordenadas: GCS WGS 84 Datum: WGS 84

Fonte: Google Maps e IBGE.

Fig. 1. Localização da área de estudo. A. Brasil; B. Estado do Maranhão; C. Praia de Panaquatira. Fonte: Lima et al. (2017).

A restinga da praia de Panaquatira possui vegetação de manguezal em trechos rebaixados da área que ficam sujeitas a alagamentos por águas estuarinas (Lima et al., 2017). A restinga do presente estudo é considerada uma das áreas de maior expressividade em relação à vegetação de restinga do Maranhão, por presentar um bom estado de conservação, quando comparada com outras áreas do litoral do Estado, apresentando uma flora com alta riqueza e diferentes tipos fisionômicos (Lima et al., 2017; Lima \& Almeida Jr., 2018).

\section{Amostragem da vegetação}

A amostragem foi realizada na fisionomia frutíceto fechado não inundável (fisionomia com aspecto fechado, que apresentam uma composição de arbustos e arvoretas se desenvolvendo muito próximos, com justaposição das copas (Lima et al., 2017). Para isso, foi utilizado o método de pontos-quadrantes (Cottam \& Curtis, 1956), onde foram instaladas cinco transecções paralelas, de $100 \mathrm{~m}$ cada. Em cada transecção foram alocados 10 pontos quadrantes, espaçados $10 \mathrm{~m}$ entre si, perfazendo o total de 50 pontos. Foram incluídos na amostragem os indivíduos lenhosos vivos com perímetro à altura do solo $(\mathrm{PAS}) \geq 10 \mathrm{~cm}$. Para cada indivíduo foi medido o perímetro do caule (com auxílio de uma fita métrica), a altura da planta (estimada com auxílio de vara graduada) e a distância da planta até o centro do quadrante. Para as palmeiras foram medidas as estipes aéreas, considerando o mesmo valor de critério de inclusão, com PAS $\geq 10 \mathrm{~cm}$; cabe destacar que na área não foi registrada nenhuma palmeira com estipe subterrânea. 
Indivíduos ramificados ao nível do solo foram considerados na amostragem quando pelo menos uma dos ramos atendia ao critério de inclusão, com PAS $\geq 10 \mathrm{~cm}$. Diante disso, o perímetro de cada ramificação era medido e depois todos os valores eram somados para ser calculado e transformado no valor correspondente ao diâmetro da planta (Moro \& Martins, 2011). Os valores dos perímetros foram convertidos em diâmetros para os cálculos dos parâmetros estruturais. Todos os arbustos, árvores e palmeiras amostrados foram etiquetados com plaquetas de PVC e numerados sequencialmente.

As espécies amostradas foram coletadas conforme técnicas usuais para plantas vasculares (Peixoto \& Maia, 2013). Em laboratório, foram identificadas a partir de referências bibliográficas especializadas (Barroso et al., 1999; Barbosa et al., 2013; Morokawa et al., 2013; Silva et al., 2014) e comparação com o acervo do Herbário do Maranhão (MAR) (Almeida Jr., 2015) da Universidade Federal do Maranhão (UFMA). A organização das famílias seguiu a classificação do APG IV (APG, 2016) e após identificação, as amostras foram depositadas, como coleção testemunho, no Herbário MAR.

\section{Análise dos dados}

A estrutura horizontal foi analisada a partir dos parâmetros fitossociológicos calculados para cada espécie amostrada. Foram determinados os parâmetros de frequência relativa (FR), densidade relativa (DR), dominância relativa (DoR), valor de cobertura (VC) e valor de importância (VI) além do índice de Shannon-Wienner (H') e equabilidade de Pielou (J), através do pacote FITOPAC 2.1 (Shepherd, 2009).

Foram construídos histogramas de distribuição dos indivíduos por classes de diâmetro, com intervalos de $10 \mathrm{~cm}$ e por classes de alturas, com intervalos de $1 \mathrm{~m}$. Esses valores de intervalos foram adotados no intuito de seguir o padrão de outros estudos realizados em áreas de restinga da região Nordeste do Brasil (Zickel et al., 2015; SantosFilho et al., 2015; Santos et al., 2015; Machado \& Almeida Jr., 2019; Almeida Jr. et al. 2020; Paiva \& Almeida Jr., 2020).

Para melhor descrição fitossociológica e conhecimento das espécies ocorrentes na restinga estudada caracterizou-se a estrutura da comunidade com base no Valor de Importância das espécies, conforme proposto por Pereira et al. (2004). De acordo com esses autores as espécies foram classificadas em dominantes (VI $>10)$, intermediárias $(10>\mathrm{VI}>1)$ e $\operatorname{raras}(\mathrm{VI}<1)$. O padrão de distribuição espacial local das espécies foi estimado segundo o índice de Morisita padronizado (Ip) proposto por Smith-Gill (1975), adaptado por Krebs (1999), que desconsidera as espécies com apenas um indivíduo. Cada espécie pode ser classificada como regular (Ip $<-0,5)$, aleatória $(-0,5<\operatorname{Ip}>0,5)$ ou agregada (Ip $>0,5)$. Assim, foi avaliado o padrão de distribuição de 13 espécies.

Para conhecimento sobre a distribuição dos táxons nos principais domínios fitogeográficos brasileiros (Amazônia, Caatinga, Cerrado, Floresta Atlântica, Pampa e Pantanal) foram utilizados os dados da Lista de espécies da Flora do Brasil (2020) e literatura especializada. Para essa análise foram considerados, apenas, os táxons identificados até epíteto específico.

\section{Resultados}

Foram identificadas 22 espécies, distribuídas em 18 gêneros e 14 famílias de plantas arbustivas e arbóreas (Tabela 1), correspondendo a 200 indivíduos amostrados. Dentre as espécies registradas, Attalea speciosa, Coccoloba ramosissima, Guettarda angelica e Himatanthus drasticus são caracterizadas como endêmicas do Brasil.

As famílias mais ricas em número de espécies foram Myrtaceae, com quatro espécies, seguida de Arecaceae, Fabaceae, Ochnaceae, Polygonaceae e Rubiaceae, com duas espécies, cada. As demais famílias apresentaram apenas uma espécie. Quanto ao número de indivíduos, Arecaceae (com 56 indivíduos), Myrtaceae (com 39), Fabaceae (36) e Apocynaceae (com 35) foram as famílias que se destacaram no arranjo estrutural da restinga, contabilizando $82,5 \%$ dos indivíduos amostrados. O índice de Shannon foi 2,401 nat.ind ${ }^{-1}$ e a equabilidade de Pielou resultou em 0,749.

Dentre as espécies com maior abundância destacaram-se Himatanthus drasticus (35), Attalea speciosa (33), Chamaecrista ramosa (33), Eugenia biflora (27) e Astrocaryum vulgare (23). Essas espécies também apresentaram destaque quanto ao Valor de Importância (VI) ecológico, porém 


\section{Belfort et al. - Vegetação lenhosa em uma restinga no litoral Amazônico}

Tabela 1. Espécies amostradas na área de restinga em Panaquatira, São José de Ribamar, Maranhão. Parâmetros fitossociológicos: Número de indivíduos (Ni); Frequência Relativa (FR); Densidade Relativa (DR); Dominância Relativa (DoR); Valor de Cobertura (VC); Valor de Importância (VI). Domínios fitogeográficos: Amazônia (AM); Caatinga (CAA); Cerrado (CR); Floresta Atlântica (FA); Pampas (PA); Pantanal (PN). Espécies ordenadas por Valor de Importância.

\begin{tabular}{|c|c|c|c|c|c|c|c|c|c|c|c|c|c|c|}
\hline Espécies & Famílias & $\begin{array}{l}\text { Voucher } \\
\text { MAR }\end{array}$ & AM & $\begin{array}{l}\text { D } \\
\text { fitog } \\
\text { CAA }\end{array}$ & $\begin{array}{l}\text { omín } \\
\text { eogr } \\
\text { CR }\end{array}$ & $\begin{array}{l}\text { hios } \\
\text { ráfico } \\
\text { FA }\end{array}$ & $\begin{array}{l}\text { OS } \\
\text { PA }\end{array}$ & PN & $\mathbf{N i}$ & $\begin{array}{l}\text { FR } \\
(\%)\end{array}$ & $\begin{array}{l}\text { DR } \\
(\%)\end{array}$ & $\begin{array}{l}\text { DoR } \\
(\%)\end{array}$ & $\begin{array}{l}\text { VC } \\
(\%)\end{array}$ & $\begin{array}{l}\text { VI } \\
(\%)\end{array}$ \\
\hline Astrocaryum vulgare Mart. & Arecaceae & 9336 & $x$ & - & $x$ & - & - & - & 23 & 12,08 & 11,5 & 37,5 & 24,5 & 20,36 \\
\hline $\begin{array}{l}\text { Attalea speciosa } \\
\text { Mart. ex Spreng. }\end{array}$ & Arecaceae & 9337 & $\mathrm{X}$ & - & $\mathrm{X}$ & - & - & - & 33 & 14,77 & 16,5 & 26,94 & 21,7 & 19,4 \\
\hline $\begin{array}{l}\text { Himatanthus drasticus } \\
\text { (Mart.) Plumel }\end{array}$ & Apocynaceae & 9328 & $x$ & $\mathrm{X}$ & $\mathrm{X}$ & - & - & - & 35 & 18,12 & 17,5 & 8,55 & 13 & 14,72 \\
\hline $\begin{array}{l}\text { Chamaecrista } \\
\text { ramosa (Vogel) H.S. } \\
\text { Irwin \& Barneby }\end{array}$ & Fabaceae & 9320 & $X$ & $X$ & $\mathrm{X}$ & $\mathrm{X}$ & - & $\mathrm{X}$ & 33 & 11,41 & 16,5 & 4,15 & 10,3 & 10,68 \\
\hline Eugenia biflora (L.) DC. & Myrtaceae & $\begin{array}{l}9325 \\
9324\end{array}$ & $\mathrm{x}$ & $\mathrm{X}$ & $x$ & - & - & - & 27 & 12,75 & 13,5 & 4,57 & 9 & 10,27 \\
\hline $\begin{array}{l}\text { Byrsonima crassifolia } \\
\text { (L.) Kunth }\end{array}$ & Malpighiaceae & 9319 & $\mathrm{X}$ & $x$ & $x$ & $\mathrm{X}$ & - & $\mathrm{X}$ & 7 & 4,7 & 3,5 & 5,8 & 4,6 & 4,66 \\
\hline Myrcia multiflora (Lam.) DC. & Myrtaceae & $\begin{array}{l}9323 \\
9332\end{array}$ & $\mathrm{x}$ & $x$ & $x$ & $\mathrm{x}$ & - & - & 8 & 4,03 & 4 & 0,91 & 2,4 & 2,98 \\
\hline Matayba guianensis Aubl. & Sapindaceae & 9731 & $x$ & - & $x$ & $x$ & - & $x$ & 7 & 4,03 & 3,5 & 0,59 & 2 & 2,7 \\
\hline $\begin{array}{l}\text { Ouratea hexasperma } \\
\text { (A.St. Hil.) Baill. }\end{array}$ & Ochnaceae & 9331 & - & - & $x$ & - & - & - & 5 & 3,36 & 2,5 & 0,32 & 1,4 & 2,05 \\
\hline Anacardium occidentale L. & Anarcardiaceae & 9317 & $x$ & $x$ & $x$ & $x$ & $x$ & $x$ & 1 & 0,67 & 0,5 & 4,66 & 2,5 & 1,94 \\
\hline $\begin{array}{l}\text { Pouteria ramiflora } \\
\text { (Mart.) Radlk. }\end{array}$ & Sapotaceae & 9726 & - & - & - & - & - & - & 1 & 0,67 & 0,5 & 4,19 & 2,3 & 1,78 \\
\hline $\begin{array}{l}\text { Andira } \\
\text { surinamensis (Bondt) } \\
\text { Splitg. ex Amshoff }\end{array}$ & Fabaceae & 9318 & $x$ & $x$ & $\mathrm{X}$ & - & - & - & 3 & 2,01 & 1,5 & 0,37 & 0,9 & 1,29 \\
\hline $\begin{array}{l}\text { Myrcia cuprea } \\
\text { (O.Berg) Kiaersk. }\end{array}$ & Myrtaceae & 9333 & $x$ & - & - & - & - & - & 3 & 2,01 & 1,5 & 0,34 & 0,9 & 1,28 \\
\hline Combretaceae & Combretaceae & 9322 & - & - & - & - & - & - & 3 & 2,01 & 1,5 & 0,15 & 0,8 & 1,22 \\
\hline $\begin{array}{l}\text { Ouratea fieldingiana } \\
\text { (Gardner) Engl. }\end{array}$ & Ochnaceae & 9330 & - & - & - & $x$ & - & - & 3 & 2,01 & 1,5 & 0,09 & 0,7 & 1,2 \\
\hline Guapira sp. & Nyctaginaceae & $\begin{array}{l}9723 \\
9728\end{array}$ & - & - & - & - & - & - & 2 & 1,34 & 1 & 0,08 & 0,5 & 0,8 \\
\hline $\begin{array}{l}\text { Eugenia stictopetala } \\
\text { Mart. ex DC. }\end{array}$ & Myrtaceae & 9326 & $x$ & $x$ & $x$ & $\mathrm{X}$ & - & - & 1 & 0,67 & 0,5 & 0,3 & 0,4 & 0,49 \\
\hline $\begin{array}{l}\text { Coccoloba } \\
\text { ramosissima Wedd. }\end{array}$ & Polygonaceae & 9732 & $\mathrm{X}$ & - & - & $x$ & - & - & 1 & 0,67 & 0,5 & 0,28 & 0,3 & 0,48 \\
\hline $\begin{array}{l}\text { Chomelia obtusa Cham. } \\
\text { \& Schltdl. }\end{array}$ & Rubiaceae & 9733 & $x$ & $\mathrm{X}$ & $x$ & $x$ & - & - & 1 & 0,67 & 0,5 & 0,11 & 0,3 & 0,42 \\
\hline $\begin{array}{l}\text { Guettarda angelica Mart. } \\
\text { ex Müll. Arg. }\end{array}$ & Rubiaceae & 9327 & - & $X$ & - & - & - & - & 1 & 0,67 & 0,5 & 0,03 & 0,2 & 0,4 \\
\hline $\begin{array}{l}\text { Protium } \\
\text { heptaphyllum (Aubl.) } \\
\text { Marchand }\end{array}$ & Burseraceae & 9329 & $x$ & $\mathrm{X}$ & $\mathrm{X}$ & $x$ & - & - & 1 & 0,67 & 0,5 & 0,03 & 0,2 & 0,4 \\
\hline Coccoloba latifolia Lam. & Polygonaceae & 9321 & $x$ & $\mathrm{X}$ & $\mathrm{X}$ & - & - & - & 1 & 0,67 & 0,5 & 0,03 & 0,2 & 0,4 \\
\hline
\end{tabular}


em uma ordem diferente, a saber: Astrocaryum vulgare (20,36\%), Attalea speciosa (19,40\%), Himatanthus drasticus (14,72\%), Chamaecrista ramosa (10,68\%), Eugenia biflora $(10,27 \%)$ e Byrsonima crassifolia (4,66\%) (Tabela 1).

As espécies de maior VI representaram mais da metade $(80,11 \%)$ do total do Valor de Importância. As espécies avaliadas como intermediárias foram representadas por 16 espécimes $(19,89 \%$ do VI). A dominância por poucas espécies indica que a comunidade estudada apresenta uma estrutura oligárquica. Astrocaryum vulgare representa a espécie mais importante devido a dominância relativa (37,50\%), assim como Attalea speciosa que apresentou dominância relativa de 26,94\%. Himatanthus drasticus foi a espécie que se destacou quanto a frequência e densidade relativa, com $18,12 \%$ e $17,50 \%$, respectivamente.

Os valores de diâmetro variaram de $3,18 \mathrm{~cm}$ a $110 \mathrm{~cm}$. O maior número de indivíduos $(73 \%)$ concentrou-se na primeira classe diamétrica, com intervalo de $3 \mathrm{~cm}$ a $13 \mathrm{~cm}$; para a segunda classe, foram registrados $16,5 \%$ do total de indivíduos
(Fig. 2). Os maiores valores diamétricos foram registrados em dois indivíduos, representados por Pouteria ramosa sp., com $110 \mathrm{~cm}$ e Astrocaryum vulgare, com $89,17 \mathrm{~cm}$. Sobre as plantas ramificadas desde a base, obteve-se um total de 41 indivíduos (20,5\%), destacando-se Eugenia biflora, Himatanthus drasticus, Chamaecrista ramosa, Myrcia multiflora, Attalea speciosa, Astrocaryum vulgare e Matayba guianensis.

A altura dos indivíduos registrados neste estudo variou de $0,3 \mathrm{~m}$ a $16 \mathrm{~m}$, com altura média de $3,16 \mathrm{~m}$. A maior frequência de altura dos indivíduos amostrados corresponde a segunda (1,1-2m) e terceira (2,1-3m) classes, com 65 e 49 indivíduos, respectivamente, totalizando $77,5 \%$ (Fig. 3). O indivíduo de menor altura foi representado por Chamaecrista ramosa, amplamente registada na área, desenvolvendo-se em grandes moitas e com grande representatividade na restinga. A última classe contemplou dois indivíduos com alturas superiores a $10 \mathrm{~m}$, um espécime de Anacardium occidentale com $15 \mathrm{~m}$ e outro espécime de Byrsonima crassifolia com $16 \mathrm{~m}$.

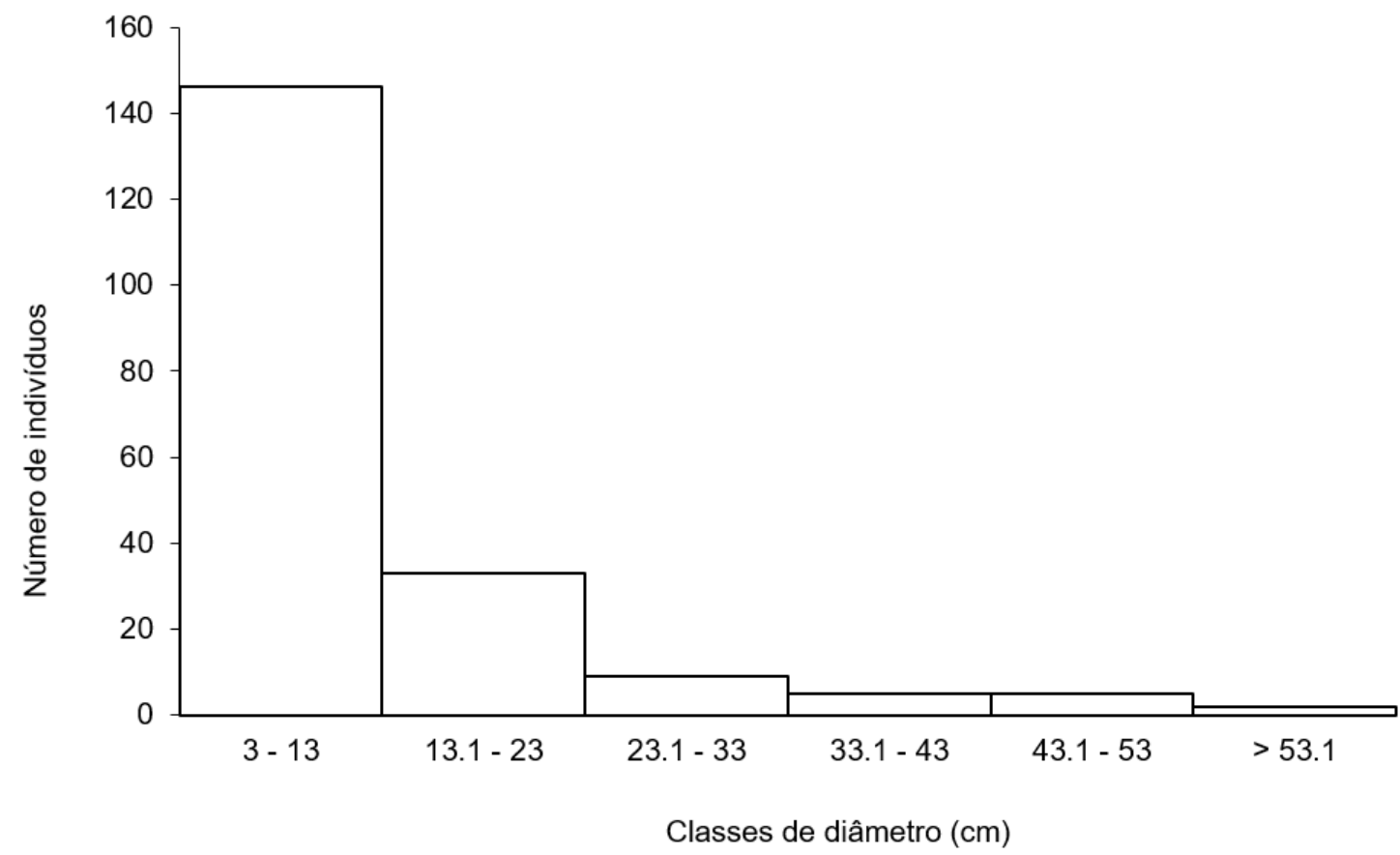

Fig. 2. Distribuição dos indivíduos arbustivos e arbóreos por classe de diâmetro $(10 \mathrm{~cm})$ na restinga de Panaquatira, São José de Ribamar, Maranhão. 


\section{Belfort et al. - Vegetação lenhosa em uma restinga no litoral Amazônico}

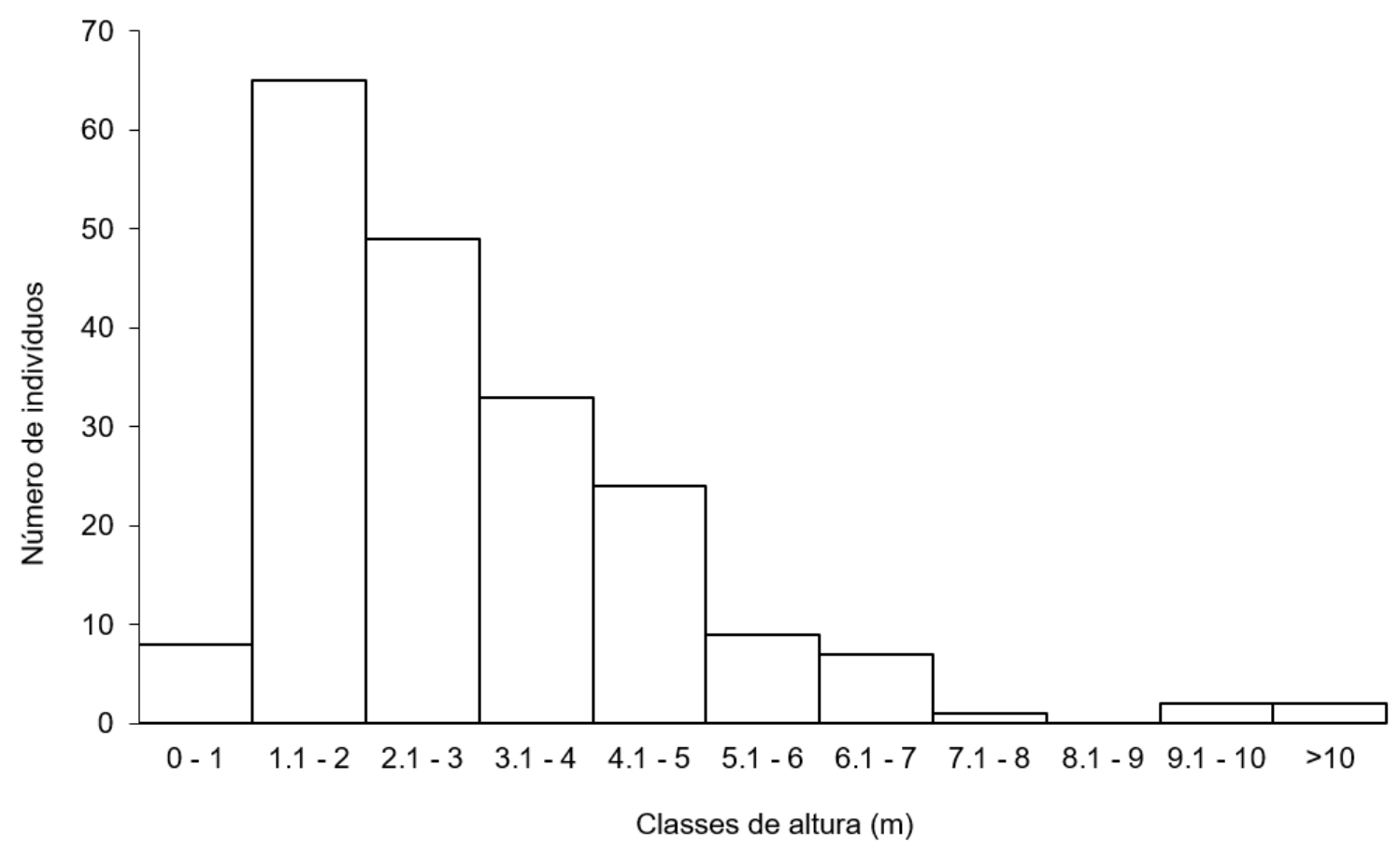

Fig. 3. Distribuição dos indivíduos arbustivos e arbóreos por classe de altura $(1 \mathrm{~m})$ na restinga de Panaquatira, São José de Ribamar, Maranhão.

Foi avaliado o padrão de distribuição local de 13 espécies, das quais 12 encontram-se distribuídas aleatoriamente na área. A única espécie com padrão de distribuição diferenciado foi Chamaecrista ramosa, apresentando distribuição do tipo agregada, com Ip igual a 0,51 . Na análise de distribuição nos principais domínios fitogeográficos brasileiros, 20 espécies foram analisadas. Deste total, Anacardium occidentale, Byrsonima crassifolia e Chamaecrista ramosa apresentaram registro em quase todos os diferentes tipos de vegetação no Brasil. Cinco espécies (Chomelia obtusa, Eugenia stictopetala, Matayba guianensis, Myrcia multiflora e Protium heptaphyllum) apresentaram registro em quatro domínios fitogeográficos e as demais espécies ocorrem em menos de três domínios fitogeográficos; destacando assim as variações quanto a amplitude de distribuição dessas espécies. A partir dos dados analisados, cerca de $84 \%$ das espécies registradas neste estudo são comuns ao domínio Amazônico, 79\% ao Cerrado, $63 \%$ a Caatinga, $52 \%$ a Mata Atlântica, $21 \%$ aos Pampas e 5\% ao Pantanal (Tabela 1). Com isso, percebe-se a influência de espécies do domínio Amazônico, Cerrado e Caatinga na colonização da restinga de Panaquatira, demonstrando a condição ecotonal, diante das particularidades quanto a paisagem, fisionomias e diversidade, e essa mistura reforça uma configuração de vegetação em mosaico para a área de estudo.

\section{DIscussão}

As famílias mais ricas registradas no presente estudo também se destacam nas restingas do litoral nordestino setentrional. No entanto, ressalta-se aqui a ausência de estudos fitossociológicos da comunidade lenhosa em áreas do litoral amazônico, impossibilitando assim comparações entre estudos que utilizaram os mesmos critérios metodológicos. Apesar disso, considerando os estudos florísticos para as restingas dos estados do Pará e Amapá, as famílias Fabaceae, Rubiaceae, Myrtaceae, Apocynaceae e Melastomataceae destacam-se como as mais representativas em número de 
espécies (Silva et al., 2010; Silva et al., 2021). Reforçando a importância desse conjunto de plantas para a colonização das áreas litorâneas do Maranhão.

A riqueza específica representada por Myrtaceae já foi constatada para diferentes áreas de restingas do litoral brasileiro (Giaretta et al., 2013; Santos et al., 2015). Em análises sobre a estrutura da vegetação lenhosa no litoral nordestino, Myrtaceae também foi registrada como a mais rica nas restingas da Bahia (Santos et al., 2015), Pernambuco (Almeida Jr. et al., 2011), Rio Grande do Norte (Medeiros et al., 2014), Alagoas (Medeiros et al., 2010) e Paraíba (Vicente et al., 2014). Essa riqueza se deve pela grande quantidade de espécies registradas no Brasil (Sobral et al., 2015). E por essas espécies se desenvolverem em diferentes tipos de ambientes, estando presente na maioria das formações vegetais (Oliveira-Filho \& Fontes, 2000). Incluindo as áreas de solos arenosos como as restingas (Rosário et al., 2005; Souza et al., 2007; Amorim \& Almeida Jr., 2021); que possuem solos ácidos, com baixa fertilidade e baixa disponibilidade hídrica (Scarano, 2002; Magnago et al., 2012; Santos-Filho et al., 2013).

Apesar de Myrtaceae ter apresentado maior riqueza específica na área amostrada, não foi a família mais abundante, assim como verificado em outros estudos ao longo do litoral brasileiro (Thomazi \& Silva, 2014; Santos-Filho et al., 2013; Santos-Filho et al., 2015; Almeida Jr. et al., 2020; Paiva \& Almeida Jr., 2020). Arecaceae foi a família que se destacou em abundância, sendo representada por Astrocaryum vulgare e Attalea speciosa. No Brasil, as espécies de Arecaceae ocorrem em praticamente todas as formações vegetais (Souza \& Lorenzi, 2012).

A diversidade encontrada $\left(2,401\right.$ nat.ind $\left.^{-1}\right)$ foi similar aos valores registrados em estudos semelhantes realizados por Santos-Filho et al. (2015) no Piauí (2.22 nat.ind $\left.{ }^{-1}\right)$, Castro et al. (2012) no Ceará (2.8 nat.ind ${ }^{-1}$ ) e por Almeida Jr. \& Zickel (2012) no Rio Grande do Norte $(2,59$ nat.ind $\left.{ }^{-1}\right)$, nas restingas do litoral nordestino setentrional. Ao analisar apenas o litoral, pode-se confirmar que as restingas da Bahia, Alagoas, Pernambuco e Paraíba apresentam maior diversidade quanto a composição lenhosa, apresentando valores de 3,553 nat.ind ${ }^{-1}$ (Santos et al., 2015), 3,330 nat.ind ${ }^{-1}$ (Medeiros et al., 2010),
3,508 nat.ind ${ }^{-1}$ (Almeida Jr. et al., 2011) e 3,018 nat.ind $^{-1}$ (Vicente et al., 2014). Considerando os estudos do Maranhão, o presente estudo $(2,401$ nat.ind ${ }^{-1}$ ) apresentou diversidade menor em relação aos que foram registrados nas restingas de Curupu (2.9 nat.ind ${ }^{-1}$ ), município de Raposa (Machado \& Almeida Jr., 2019), Itatinga (2.92 nat. ind ${ }^{-1}$ ), em Alcântara (Almeida Jr. et al., 2020) e na restinga da praia da Guia (3.03 nat.ind ${ }^{-1}$ ), em São Luís (Paiva \& Almeida Jr., 2020). Os valores de diversidade para essas regiões pode estar relacionada a quantidade de espécies raras amostradas (Felfili \& Rezende, 2003) devido ao maior valor que o índice atribui a esse grupo de plantas ou devido as particularidades de cada área.

O valor obtido para a equabilidade (J' de 0,749 ) pode representar uma área com pouca agregação de espécies e distribuição aleatória da maioria dos indivíduos na área (Medeiros et al., 2010). O padrão pode ser uma resposta às interações intra e interespecíficas, como competição, predação, dispersão, crescimento, reprodução entre outras características.

E diante das particularidades observadas no Maranhão, devido aos ambientes ecotonais, os valores do H' encontrados representam uma alta diversidade para as restingas; por se tratar de um ecossistema com baixa disponibilidade hídrica, escassez de nutrientes no solo, alta salinidade, fortes ventos que também podem estar associados a outros fatores como inundações, queimadas, pastagens, supressão da vegetação, construções imobiliárias e até o pisoteio (causados pelo fluxo de pessoas caminhando no ambiente) (SantosFilho et al., 2013; Paiva \& Almeida Jr., 2020).

$\mathrm{O}$ teste de Morisita confirmou que as espécies se desenvolvem aleatoriamente, devido a organização espacial das plantas acontecerem de forma independente no ambiente (Araújo et al., 2014). Essa distribuição pode estar relacionada ao tipo de agente dispersor (Jorge et al., 2015), nesse caso, o vento, que pode ser considerado como mais eficiente para áreas litorâneas; e também estar relacionada aos padrões estocásticos de estabelecimento, crescimento/desenvolvimento e competição das espécies na restinga do presente estudo. Com isso, a predominância de espécies com distribuição espacial aleatória destaca uma homogeneidade no ambiente, permitindo assim que sejam analisadas quais espécies, a partir do 


\section{Belfort et al. - Vegetação lenhosa em uma restinga no litoral Amazônico}

número de indivíduos, podem ser "exploradas" ou mantidos na área, de forma adequada e direcionada para que seja garantida a reprodução dessas espécies.

Chamaecrista ramosa foi uma exceção na amostragem, pois apresentou padrão de distribuição agregado. Esse tipo de distribuição ocorre geralmente em espécies autocóricas, por depositarem as sementes próximos à planta mãe, estabelecendo o padrão agregado (Araújo et al., 2014). Além disso, esse padrão pode estar associado a ocorrência de diferentes microhábitats e condições edáficas (Lima \& Prado, 2007). A dispersão de espécies de Fabaceae (como o gênero Chamaecrista) em campos rupestres, por exemplo, ocorrem mesmo com ventos fortes e ar mais seco (Dutra et al., 2009); condições ambientais semelhantes as quais Chamaecrista ramosa está submetida na restinga estudada, explicando assim o maior destaque dessa população na área. Cabe destacar, também, que espécies que apresentam reprodução vegetativa, pode ter uma tendência à formação de padrões agregados; visto que os indivíduos se desenvolveriam mais próximos entre si.

As espécies Astrocaryum vulgare, Attalea speciosa, Himatanthus drasticus, Chamaecrista ramosa, Eugenia biflora e Byrsonima crassifolia foram as que mais contribuíram, quanto ao valor de importância, no arranjo estrutural da restinga ecotonal. E Attalea speciosa, Coccoloba ramosissima, Guettarda angelica e Himatanthus drasticus são consideradas endêmicas do Brasil (Flora do Brasil, 2020), destacando a importância de conservação dessa restinga ecotonal.

Astrocaryum vulgare é uma planta pioneira e sua abundância na área pode estar relacionada a capacidade de desenvolvimento em solos pobres e/ou excesso de perfilhos que facilitam a regeneração (Shanley \& Medina, 2005). Attalea speciosa também é considerada uma espécie pioneira e uma colonizadora eficiente (Ribeiro \& Walter, 1998; Mitja \& Ferraz, 2001) de ambientes arenosos. Himatanthus drasticus destaca-se por sua capacidade de rebrota a partir das raízes (Moro et al., 2011), o que contribui para seu desenvolvimentos em áreas de restinga.

No Maranhão, Machado \& Almeida Jr. (2019) ao analisarem o estrato lenhoso de uma restinga em uma região de transição entre os litorais amazônico e nordestino, destacaram Astrocaryum vulgare e Eugenia biflora como as de maior VI. Essa semelhança justifica-se pela proximidade geográfica e por ambas as áreas pertencerem a mesma região ecotonal. Diferente dos estudos realizados em restingas do litoral nordestino (Almeida Jr. et al., 2011; Cantarelli et al., 2012; Santos et al., 2015; Zickel et al., 2015), que não registraram nenhuma das espécies de maior VI catalogadas no presente estudo. Isso demonstra as diferenças estruturais e as distintas influências recebidas pela vegetação, considerando o componente lenhoso ao longo do litoral nordestino.

Quanto a caracterização da estrutura da comunidade com base no VI (Pereira et al., 2004), foram observadas poucas espécies dominantes. Isso se deve, provavelmente, aos fatores adversos aos quais a comunidade está submetida (Scarano, 2009), como solo arenoso de baixa fertilidade, escassez de água, alta salinidade, intensa ação do vento, comuns ao ecossistema de restinga (Castro et al., 2012; Menezes et al., 2012; Medeiros et al., 2014). O estabelecimento de espécies dominantes pode ter possibilitado condições para o desenvolvimento de espécies menos resistentes às condições ambientais extremas das restingas (Araújo et al., 2016).

As distribuições de diâmetro e altura do presente estudo mostraram que a comunidade é dominada por indivíduos jovens de baixo porte e com troncos finos, semelhante vegetação de restinga de Curupu (Machado \& Almeida Jr., 2019). A distribuição diamétrica decrescente, em forma de J-invertido, caracteriza uma comunidade em equilíbrio entre mortalidade e recrutamento dos indivíduos (Rodrigues \& Magalhães, 2011). Os maiores valores de diâmetro foram registrados em um indivíduo de grande porte, representado por Pouteria ramosa $(110 \mathrm{~cm})$, e um indivíduo de muitas ramificações, representado por Astrocaryum vulgare $(89,17 \mathrm{~cm})$. A quantidade de indivíduos ramificados pode ter relação com a capacidade de rebrota de cada espécie, sendo uma estratégia de crescimento, diante das particularidades das áreas de restinga.

Em relação aos domínios fitogeográficos, a espécie Anacardium occidentale foi a única com registros para todos os domínios. Esse fato pode estar relacionado a distribuição dessa espécie em regiões tropicais e subtropicais (Souza \& Lorenzi, 2012), por ser nativa e de grande importância 
econômica (Araújo et al., 2010), sendo mantidas nas áreas litorâneas onde já existem ou sendo inseridas e cultivadas, onde não se encontrava de forma natural, devido ao potencial econômico do fruto (Zickel et al., 2015). Entre as espécies amostradas, nenhuma é restrita a um único bioma, assim como não há registro de endemismo. E por estar situada em uma área ecotonal do litoral maranhense, muitas espécies são comuns aos domínios Amazônico e Cerrado, porém com maior proporção de plantas Amazônicas; conferindo maior singularidade a essas restingas.

\section{Conclusão}

A estrutura da restinga entre os litorais amazônico e nordestino mostra que a área apresenta um valor de diversidade que deve ser considerado alto por ser tratar de área ecotonal. E apresentaram valores de diversidade similares a outras áreas de restingas do nordeste. A vegetação estudada mostra uma estrutura oligárquica, dominada por indivíduos jovens de porte baixo e calibres finos, representando uma comunidade com espécies advindas da floresta amazônica e do cerrado, cujas particularidades reforçam a necessidade de conservação.

Apesar da ausência de estudos com os mesmos critérios metodológicos, impossibilitando comparações com as restingas do litoral amazônico, as famílias registradas no presente estudo também se destacaram na composição florística das áreas da costa amazônica e da costa nordestina. Isso se deve a facilidade de desenvolvimento das espécies dessas famílias diante das condições limitantes dos ecossistemas litorâneos. A análise do valor de importância indica possíveis diferenças estruturais entre a restinga ecotonal da costa maranhense com as restingas do litoral nordestino. No entanto, faz-se necessário mais estudos nas áreas ecotonais para elucidar questões referentes ao arranjo estrutural e distribuição das espécies nas restingas da costa do Maranhão.

\section{CONTRIBUIÇÃo dOS AUTORES}

LF contribuição com a coleta de dados, análise e interpretação dos dados, elaboração do manuscrito. FRFN revisão crítica, adição de conteúdo intelectual e supervisão geral. EBAJ contribuição com a conceituação o desenho experimental, revisão crítica, adição de conteúdo intelectual e supervisão geral.

\section{Agradecimentos}

Os autores agradecem a Fundação de Amparo à Pesquisa e ao Desenvolvimento Científico e Tecnológico do Maranhão - FAPEMA pelo financiamento do projeto. À Coordenação de Aperfeiçoamento de Pessoal de Nível Superior CAPES (finance code 001). Ao CNPq e ao FINEP. A Universidade Federal do Maranhão (UFMA) e ao Laboratório de Estudos Botânicos (LEB) pela estrutura física e recursos humanos, em especial a Ariade Silva, Bruna Freire, Gustavo Lima e Ana Cássia Medeiros.

\section{Bibliografia}

ALMEIDA JR, E.B., SANTOS-FILHO, F.S., ARAÚJO, E.L. \& ZICKEL, C.S. 2011. Structural characterization of the woody plants in restinga of Brazil. J. Ecol. Nat. Environ. 3: 95-103. https://doi.org/10.5897/JENE.9000098

ALMEIDA JR., E.B. \& ZICKEL, C.S. 2012. Análise fitossociológica do estrato arbustivo-arbóreo de uma floresta de restinga no Rio Grande do Norte. Agrária 7: 286-291.

https://doi.org/10.5039/agraria.v7i2a1218

ALMEIDA JR., E.B. 2015. Herbário do Maranhão, Maranhão (MAR). U. BioSci. 4: 129-132.

ALMEIDA JR., E.B., CORREIA, B.E.F. \& SANTOSFILHO, F.S. 2020. Diversity and structure of the woody component of a restinga in Alcântara, Maranhão State, Brazil. Acta Brasiliensis 4: 85-90. https://doi.org/10.22571/2526-4338261

AMORIM, G.S. \& ALMEIDA JR., E.B. 2021. A família Myrtaceae nas restingas da Ilha do Maranhão, Brasil. Iheringia. Sér. Bot. 76: e2021008.

ANGIOSPERM PHYLOGENY GROUP (APG) IV. 2016. An update of the Angiosperm Phylogeny Group classification for the orders and families of flowering plants: APG IV. Bot. J. Linn. Soc. 181: 1-20. https://doi.org/10.1111/boj.12385

ARAUJO, A.C.M., SILVA, A.N.F. \& ALMEIDA JR., E.B. 2016. Caracterização estrutural e status de 


\section{Belfort et al. - Vegetação lenhosa em uma restinga no litoral Amazônico}

conservação do estrato herbáceo de dunas da Praia de São Marcos, Maranhão, Brasil. Acta Amaz. 46: 247-258.

https://doi.org/10.1590/1809-4392201504265

ARAÚJO, D.C.D., TARSITANO, M.A.A., COSTA, T.V.D. \& RAPASSI, R.M.A. 2010. Análise técnica e econômica do cultivo do cajueiro-anão (Anacardium occidentale L.) na regional de Jales-SP. Rev Bras Frutic 32: 444-450.

ARAUJO, D.S.D. \& HENRIQUES, R.P.B. 1984. Análise florística das restingas do estado do Rio de Janeiro. In: LACERDA, L.D., ARAÚJO, D.S.D., CERQUEIRA, R. \& TURQ, B. (Org.). Restingas: Origem, Estrutura e Processos. Niterói: CEUFF.

ARAÚJO, E.J.G., DAVID, H.C., PÉLlICO NETTO, S., MORAIS, V.A., \& SCOLFORO, J.R.S. 2014. Padrão espacial de espécies arbóreas em fragmento de floresta estacional semidecidual. Rev. Cienc. Agrar. 57: 166-171.

BARBOSA, C.V.O., BASTOS, M.N.C., SOUSA, J.S. \& CRUZ, A.P.O. 2013. Estudo taxonômico de Sapindaceae do litoral Paraense. Rodriguésia 64: 807-815. https://doi.org/10.1590/S2175-78602013000400010

BARROSO, G.M., MORIN, M.P., PEIXOTO, A.L. \& ICHOSO, C.L.F. 1999. Frutos e Sementes Morfologia Aplicada à Sistemática de Dicotiledôneas, Viçosa, Editora UFV.

BRAZ, D.M., JACQUES, E.L., SOMNER, G.V., SYLVESTRE, L.S., ROSA, M.M.T., PEREIRAMOURA, M.V.L. et al. 2013. Restinga de Praia das Neves, ES, Brasil: caracterização fitofisionômica, florística e conservação. Biota Neotrop. 13: 315-331.

CABRAL-FREIRE, M.C. \& MONTEIRO, R. 1993. Florística das praias da Ilha de São Luiz, Estado do Maranhão (Brasil): Diversidade de espécies e suas ocorrências no litoral brasileiro. Acta Amaz. 23: 125-140.

CANTARELLI, J.R.R., ALMEIDA JR., E.B., SANTOSFILHO, F.S. \& ZICKEL, C.S. 2012. Descrição da estrutura de uma vegetação de restinga da Área de Proteção Ambiental (APA) de Guadalupe, litoral sul de Pernambuco. Pp. 49-68. In: A.C.A. EL-DEIR, G.J.B. MOURA, E.L. ARAÚJO (Orgs.). Ecologia e conservação de ecossistemas no Nordeste do Brasil. Recife, PE: NUPEEA.

CASTRO, A.S.F., MORO, M.F. \& MENEZES, M.O.T. 2012. O complexo vegetacional da zona litorânea no Ceará: Pecém, São Gonçalo do Amarante. Acta Bot. Bras. 26: 108-124.
https://doi.org/10.1590/S0102-33062012000100013

CORREIA, B.E.F., MACHADO, M.A. \& ALMEIDA JR., E.B. 2020. Lista florística e formas de vida da vegetação de uma restinga em Alcântara, litoral ocidental do Maranhão, Nordeste do Brasil. Rev. Bras. Geogr. Fís. 13: 2198-2211.

https://doi.org/10.26848/rbgf.v13.5.p2198-2211

COTTAM, G. \& CURTIS, J. 1956. The Use of Distance Measures in Phytosociological Sampling. Ecology 37: $451-460$.

DUTRA, V.F., VIEIRA, M.F., GARCIA, F.C.P. \& LIMA, H.C. 2009. Fenologia reprodutiva, síndromes de polinização e dispersão em espécies de Leguminosae dos campos rupestres do Parque Estadual do Itacolomi, Minas Gerais, Brasil. Rodriguésia 60: 371 387. https://doi.org/10.1590/2175-7860200960210

FELFILI, J.M. \& REZENDE, R.P. 2003. Conceitos $e$ métodos em fitossociologia. Brasília: Ed. UNB.

Flora do Brasil 2020. Jardim Botânico do Rio de Janeiro [online]. Disponível em: http://floradobrasil.jbrj.gov. br. [Acesso em 17 jun. 2021].

GIARETTA, A., MENEZES, L.F.T. \& PEREIRA, O.J. 2013. Structure and floristic pattern of coastal dunes in southeastern Brazil. Acta Bot. Bras. 27: 87-107. https://doi.org/10.1590/S0102-33062013000100011

GUTERRES, A.V.F., AMORIM, I.F.F., SILVA, A.F.C. \& ALMEIDA JR., E.B. 2020. Levantamento florístico e fisionômico da restinga da praia da Guia, São Luís, Maranhão. Biodiversidade 19: 57-72.

HIRUMA, S.T., SALLUN, A.E.M., RIBEIRO, R.R. \& AZEVEDO SOBRINHO, J.M. 2008. Restinga: Conceitos e empregos do termo no Brasil e implicações na Legislação Ambiental. São Paulo: Instituto Geológico.

IMESC. 2011. Instituto Maranhense de Estudos Socioeconômicos e Cartográficos. Situação Ambiental da Ilha de São Luís. São Luís.

FLORA DO BRASIL. 2020. Rio de Janeiro: Jardim Botânico do Rio de Janeiro; 2014 [online]. .Disponível em: http://floradobrasil.jbrj.gov.br. [Acesso em abr 1 2021]

KREBS, C.J. 1999. Ecological methodology. 2.ed. New York: Benjamin/Cummings.

LIMA, R.M.S. \& PRADO, E.C. 2007. Distribuição espacial de uma população de Vernonia aurea mart. ex dc. (Asteraceae) em um fragmento de cerradão no Município de Caiapônia, GO, Brasil. Rev. Biociências. J. 23: 81-89.

LIMA, G.P., LACERDA, D.M.A., LIMA, H.P. \& ALMEIDA JR., E.B. 2017. Caracterização 
fitofisionômica da Restinga da Praia de Panaquatira, São José de Ribamar, Maranhão. Rev. Bras. Geogr. Fís. 10: 1910-1920.

https://doi.org/10.26848/rbgf.v10.6.p1910-1920

LIMA, G.P. \& ALMEIDA JR., E.B. 2018. Diversidade e similaridade florística de uma restinga ecotonal no Maranhão, Nordeste do Brasil. Interciência 43(4): 275-282.

MACHADO, M.A. \& ALMEIDA JR., E.B. 2019. Spatial structure, diversity, and edaphic factors of an area of Amazonian coast vegetation in Brazil. J. Torrey Bot. Soc. 146: 58-68. https://doi.org/10.3159/TORREY-D-18-00025.1

MAGNAGO, L.F.S., MARTINS, S.V., SCHAEFER, C.E.G.R. \& NERI, A.V. 2012. Restinga forests of the Brazilian coast: richness and abundance of tree species on different soils. An. Acad. Bras. Cienc. 84: 807-822. https://doi.org/10.1590/S0001-37652012000300023

MARTINS, P.J., MAZON, J.A., MARTINKOSKI, L., BENIN, C.C. \& WATZLAWICK, L.F. 2017. Dinâmica da vegetação arbórea em floresta ombrófila mista montana antropizada. Flor Amb. 24: e00097014.

https://doi.org/10.1590/2179-8087.097014

MEDEIROS, D.P.W., ALMEIDA JR., E.B., ABREU, M.C., SANTOS-FILHO, F.S. \& ZICKEL, C.S. 2014. Riqueza e caracterização da estrutura lenhosa da vegetação de restinga de Baia Formosa, RN, Brasil. Pesquisas Botânica 65: 183-199.

MEDEIROS, D.P.W., SANTOS-FILHO, F.S., ALMEIDA JR., E.B., PIMENTEL, R.M.M. \& ZICKEL, C.S. 2010. Estrutura do Componente Lenhoso de uma Restinga no Litoral Sul de Alagoas, Nordeste, Brasil. Rev. Bras. Geogr. Fís. 3: 146-150.

https://doi.org/10.26848/rbgf.v3i3.232647

MENEZES, C.M., AGUIAR, L.G.P.A., ESPINHEIRA, M.J.C.L. \& SILVA, V.I.S. 2009. Florística e fitossociologia do componente arbóreo do município de Conde, Bahia, Brasil. Rev. Bioc. 15: 44-55.

MENEZES, C.M., SANTANA, F.D., SILVA, V.S., SILVA, V.I.S. \& ARAUJO, D.S.D. 2012. Florística e fitossociologia em um trecho de restinga no Litoral Norte do Estado da Bahia. Biotemas 25: 31-38. https://doi.org/10.5007/2175-7925.2012v25n1p31

MITJA, D. \& FERRAZ, I. 2001. Establishment of Babassu in pastures in Pará, Brazil. Palms 45: 138-147.

MORO, M.F., CASTRO, A.S.F. \& ARAÚJO, F.S. 2011. Composição florística e estrutura de um fragmento de vegetação savânica sobre os tabuleiros pré- litorâneos na zona urbana de Fortaleza, Ceará. Rodriguésia 62: 407-423.

https://doi.org/10.1590/2175-7860201162214

MORO, M.F. \& MARTINS, F.R. 2011. Métodos de levantamento do componente arbóreo-arbustivo. In: FELFILI, J.M., EISENLOHR, P.V., MELO, M.M.R.F., ANDRADE, L.A. \& MEIRA NETO, J.A.A. (Org.). Fitossociologia no Brasil: métodos e estudos de casos. Viçosa, MG: Ed. UFV, $1^{\mathrm{a} e d}$.

MOROKAWA, R., SIMÕES, A.O. \& KINOSHITA, L.S. 2013. Apocynaceae s. str. do Parque Nacional da Serra da Canastra, Minas Gerais, Brasil. Rodriguésia 64: 179-199.

https://doi.org/10.1590/S2175-78602013000100015

PAIVA, B.H.I. \& ALMEIDA JR., E.B. 2020. Diversidade, análise estrutural e serviços ecossistêmicos da vegetação lenhosa da restinga da praia da Guia, São Luís, Maranhão, Brasil. Biodiversidade 19: 46-60.

PEEL, M.C., FINLAYSON, B.L. \& MCMAHON, T.A. 2007. Updated world map of the Köppen-Geiger climate classification. Hydrol. Earth Syst. Sci. 11: 1633-1644. https://doi.org/10.5194/hess-11-1633-2007

PEREIRA, M.C.A., CORDEIRO, S.Z. \& ARAUJO, D.S.D. 2004. Estrutura do estrato herbáceo na formação aberta de Clusia do Parque Nacional da Restinga de Jurubatiba, RJ, Brasil. Acta Bot. Bras. 18: 677-687.

https://doi.org/10.1590/S0102-33062004000300025

PEIXOTO, A.L. \& MAIA, L.C. 2013. Manual de Procedimentos para herbários. INCT-Herbário virtual para a Flora e os Fungos. Editora Universitária UFPE, Recife.

RAMOS, G.Q., COTTA, E.A. \& FONSECA-FILHO, H.D. 2016. Análise Morfológica das Folhas de Anacardium occidentale L. Biota Amaz. 6: 16-19. https://doi.org/10.18561/2179-5746/biotaamazonia. v6n $1 \mathrm{p} \% 25 \mathrm{p}$

RIBEIRO, J.F. \& WALTER, B.M.T. 1998. Fitofisionomias do bioma Cerrado. In: Sano SM, Almeida SP. (eds.) Cerrado: ambiente e flora. Planaltina, Embrapa.

ROCHA, C.F.D., BERGALLO, H.G., VAN SLUYS, M., ALVES, M.A.S. \& JAMEL, C.E. 2007. The remnants of restinga habitats in the Brazilian Atlantic Forest of Rio de Janeiro state, Brazil: habitat loss and risk of disappearance. Braz. J. Biol. 67: 263-273. https://doi.org/10.1590/S1519-69842007000200011

RODRIGUES, R.M.M. \& MAGALHÃES, L.M.S. 2011. Estrutura e florística de fragmento de floresta secundária na planície aluvionar do Rio Guandu, em Seropédica-RJ. Flor. Amb. 18: 324-333. 


\section{Belfort et al. - Vegetação lenhosa em uma restinga no litoral Amazônico}

https://doi.org/10.4322/floram.2011.052

ROSÁRIO, A.S., SECCO, R.S, AMARAL, D.D., SANTOS, J.U.M., BASTOS, M.N.C. 2005. Flórula fanerogâmica das restingas do estado do Pará. Ilhas de Algodoal e Maiandeua-2. Myrtaceae A.L. de Jussieu. Boletim do Museu Paraense Emilio Goeldi. Série Ciências Naturais 1: 31-42.

SANTOS, A.V., ALMEIDA JR., E.B., SANTOSFILHO, F.S. \& ZICKEL, C.S. 2014. Composição estrutural da vegetação lenhosa da restinga de Cabedelo, Paraíba. Revista de Geografia 31: 183-196.

SANTOS, L.M., LENZA, E., SANTOS, J.O., MARIMON, B.S., EISENLOHR, P.V., MARIMON JUNIOR, B.H. \& FELDPAUSCH, T.R. 2015. Diversity, floristic composition, and structure of the woody vegetation of the Cerrado in the CerradoAmazon transition zone in Mato Grosso, Brazil. Braz. J. Bot. 38: 877-887.

SANTOS, V.J., ZICKEL, C.S. \& ALMEIDA JR., E.B. 2015. Composição estrutural do estrato arbustivoarbóreo de uma floresta de restinga no sul da Bahia, Brasil. Pesquisas Botânica 68: 257-269.

SANTOS-FILHO, F.S., ALMEIDA JR., E.B., SOARES, C.J.R.S. \& ZICKEL, C.S. 2015. Flora and woody vegetation structure in an Insular area of restinga in Brazil. Int. J. Ecol. Environ. Sci. 41: 147-160.

SANTOS-FILHO, F.S., ALMEIDA JR., E.B. \& ZICKEL, C.S. 2013. Do edaphic aspects alter vegetation structures in the Brazilian restinga? Acta Bot. Bras. 27: 613-623.

https://doi.org/10.1590/S0102-33062013000300019

SCARANO, F.R. 2009. Plant communities at the periphery of the Atlantic rain forest: Rare-species bias and its risks for conservation. Biol. Conserv 142: 12011208. https://doi.org/10.1016/j.biocon.2009.02.027

SCARANO, F.R. 2002. Structure, Function and Floristic Relationships of Plant Communities in Stressful Habitats Marginal to the Brazilian Atlantic Rainforest. Ann. Bot. 90: 517-524.

https://doi.org/10.1093/aob/mcf189

SERRA, F.C.V., LIMA, P.B. \& ALMEIDA JR., E.B. 2016. Species richness in restinga vegetation on the eastern Maranhão State, Northeastern Brazil. Acta Amaz. 46: 271-280.

https://doi.org/10.1590/1809-4392201504704

SHANLEY, P.G. \& MEDINA, G. 2005. Frutíferas e plantas úteis na vida amazônica. CIFOR: Imazon, Belém.

SHEPHERD, G.J. 2009. Fitopac 2: manual do usuário
Programa Fitopac 2: Campinas. UNICAMP.

SILVA, R.M., MEHLIG, U., SANTOS, J.U.M., \& MENEZES, M.P.M. 2010. The coastal restinga vegetation of Pará, Brazilian Amazon: a synthesis. Rev. Bras. Bot.33: 563-573.

https://doi.org/10.1590/S0100-84042010000400005

SILVA, V.I.S. \& MENEZES, C.M. 2012. Contribuição para o conhecimento da vegetação de restinga de Massarandupió, Município de Entre Rios, BA, Brasil. Rev. Gestão Cost. Integ. 12: 239-251.

SILVA, W.L.S., ROCHA, A.E. \& SANTOS, J.U.M. 2014. Leguminosae em Savanas do Estuário Amazônico Brasileiro. Rodriguésia 65: 329-353. https://doi.org/10.1590/S2175-78602014000200004

SILVA, W.L.S., SILVA, M.F., AMARAL, D.D., CARMO, M.N.L., GURGEL, E.S.C. \& SANTOS, J.U.M. 2021. Checklist of Angiosperms in the Restingas of Pará state, Brazil, with comments on floristic affinities and phytophysiognomies. Rodriguésia 72: e01532019. https://doi.org/10.1590/2175-7860202172021

SILVEIRA, J.D. 1964. Morfologia do Litoral. In: AZEVEDO A. (Org.). Brasil: a terra e o homem. São Paulo: Cia. Editora Nacional.

SOBRAL, M., PROENÇA, C., MAZINE, M. \& LUCAS, E. 2017. Myrtaceae in Lista de espécies da flora do Brasil. Jardim Botânico do Rio de Janeiro. Disponível em http://floradobrasil.jbrj.gov.br/ (acesso em 14 nov. 2017).

SOLÓRZANO, A., PINTO, J.R.R., FELFILI, J.M. \& HAY, J.D.V. 2012. Perfil florístico e estrutural do componente lenhoso em seis áreas de cerradão ao longo do bioma Cerrado. Acta Bot. Bras. 26: 328-341.

https://doi.org/10.1590/S0102-33062012000200009

SOUZA, U.V., PEREIRA, M., SILVA, A. \& FEITOSA, A. 2006. Caracterização geomorfológica e costeira de Panaquatira, município de São José de Ribamar - MA, 2006. Disponível em: <http://www.labogef. iesa.ufg.br/links/sinageo/articles/255.pdf $>$. Acesso em: 10 dez. 2016.

SOUZA, M.C., MORIM, M.P., CONDE, M.M.S. \& MENEZES, L.F.T. 2007. Subtribo Myrciinae O. Berg (Myrtaceae) na Restinga da Marambaia, RJ, Brasil. Acta Bot. Bras. 21: 49-63. https://doi.org/10.1590/S0102-33062007000100006 SOUZA, V.C. \& LORENZI, H. 2012. Botânica Sistemática: guia ilustrado para identificação das famílias de Fanerógamas nativas e exóticas no Brasil, baseado em APG III. $3^{\mathrm{a}}$ ed. Instituto 
Plantarum, Nova Odessa, São Paulo.

SUGUIO, K., TESSLER, M.G. 1984. Planícies de cordões litorâneos quaternários do Brasil: origem e nomenclatura. In: LACERDA LD, ARAÚJO DSD, CERQUEIRA R, TURCQ B. (orgs.). Restingas: origem, estrutura e processos. Niterói - RJ: CEUFF.

THOMAZI, R.D. \& SILVA, A.G. 2014. Florística, diversidade e estrutura horizontal e vertical de uma área de vegetação arbustiva aberta numa planície arenosa costeira do Espírito Santo, sudeste do Brasil. Natureza on line 12: 10-18.

VELAZCO, S.J.E., GALVÃO, F., KELLER, H.A. \& BEDRIJ, N.A. 2015. Florística e fitossociologia de uma Floresta Estacional Semidecidual, Reserva Privada Osununú-Misiones, Argentina. Flor. Amb. 22: 1-12. https://doi.org/10.1590/2179-8087.038513

VICENTE, A., ALMEIDA JR., E.B., SANTOS-FILHO, F.S. \& ZICKEL, C.S. 2014. Composição estrutural da vegetação lenhosa da restinga de Cabedelo, Paraíba. Revista de Geografia 31: 183-196.

WALTER, B.M.T. et al. 2015. Fitofisionomias do cerrado: classificação, métodos e amostragens fitossociológicas. In: Eisenlohr, P.V., Felfili, J.M., Melo, M.M.R.F., Andrade, L.A. \& Meira-Neto, J.A.A. (Org.). Fitossociologia no Brasil: métodos e estudos de casos. Viçosa, MG: Ed. UFV, $2^{a}$ ed.

VILLWOCK, J.A., LESSA, G.C., ÂNGULO, R.J. \& DILLENBURG, S.R. 2005 Geologia e geomorfologia de regioes costeiras. In: SOUZA, C.R.G., SUGUIO, K., OLIVEIRA A.M.S. \& OLIVEIRA, P.E. (Eds). Quaternário do Brasil. Holos Editora, Ribeirão Preto: 94-113

ZICKEL, C.S., VICENTE, A., ALMEIDA JR., E.B., CANTARELLI, J.R.R. \& SACRAMENTO, A.C. 2004. Flora e Vegetação das Restingas do Nordeste Brasileiro. In: ESKINAZI-LEÇA E., NEUMANNLEITÃO S., COSTA M.F. (Orgs.). Oceanografia Um cenário tropical. Recife: Ed. Bagaço.

ZICKEL, C.S., VICENTE, A., SILVA, S.S.L., SANTOSFILHO, F.S., SOARES, C.J.R.S. \& ALMEIDA JR., E.B. 2015. Vegetação lenhosa de uma restinga em Pernambuco: descrição estrutural e similaridade. Pesquisas Botânica 68: 271-285. 